\title{
GERENCIAMENTO DE RESULTADOS E RATING DE CRÉDITO EM COMPANHIAS BRASILEIRAS DE CAPITAL ABERTO
}

\section{EARNINGS MANAGEMENT AND CREDIT RATING IN BRAZILIAN PUBLICLY TRADED COMPANIES}

0 artigo foi aprovado e apresentado no XXI Seminários em Administração (SemeAd), realizado na Universidade de São Paulo de 07/11 a 09/11 de 2018, em São Paulo (SP).

\section{RESUMO}

A obtenção de ratings mais favoráveis e a neutralização de possíveis situações de insolvência são alguns dos motivos para o gerenciamento de resultados, uma vez que as agências utilizam dados contábeis para emissão de tais notas. A proposta deste estudo é investigar o gerenciamento de resultados em empresas brasileiras com objetivo em conturbar as percepções das agências classificadoras de riscos - S\&P, Fitch Ratings e Moody's - influenciando assim, as notas de rating. Utilizou-se o modelo Kothari, Leone e Wasley (2005) para a mensuração de gerenciamento de resultados (GR), ao passo que o método Logit binário para dados em painel foi empregado para verificar a relação proposta. Os resultados demonstraram significância ao nível 10\% e uma relação inversa entre os accruals discricionários e os ratings, rejeitando-se a hipótese de que há relação positiva entre o GR e as notas de rating. Tais achados contrariam os estudos de Alissa et al. (2013) e Demirtas e Cornaggia (2013). Infere-se, portanto, que na interpretação do GR pelos analistas, a redução dos ratings ocorre como um fator de risco de solvência.

Palavras-chave: Rating. Gerenciamento de Resultados. Agências de Risco.

\begin{abstract}
Obtaining favorable ratings and the counteraction of possible insolvency situations are some of the reasons for earnings management, since agencies use accounting data to issue such notes. This study investigates the earnings management in Brazilian companies which aim to interfere with the risk rating agencies' perceptions - S\&P, Fitch Ratings and Moody's - influencing, thus, the rating notes. The Kothari, Leone and Wasley (2005) model was used to measure the earnings management (EM), whereas to verify the proposed relation the Logit binary method for panel data was applied. Our findings have shown a statistical significance of $10 \%$ and an inverse relation between discretionary accruals and ratings, rejecting the hypothesis of a positive relation between EM and rating notes. Such findings contradict the studies of Alissa et al. (2013) e Demirtas e Cornaggia (2013). Therefore, from these facts we can infer that the earnings management interpretation carried out by analysts, the reduction of the ratings occurs as a solvency risk factor
\end{abstract}

Keywords: Rating. Earnings Management. Risk Agencies.

\section{Marcela Sibim}

Doutoranda em Contabilidade Financeira e Finanças pela Universidade Federal do Paraná (UFPR). Mestre em Contabilidade Financeira e Finanças pela Universidade Federal do Paraná (UFPR). Graduada em Ciências Contábeis pela Universidade Estadual de Maringá (UEM). E-mail: marcela.sibim@gmail.com.

Paula Pontes de Campos-Rasera Doutoranda em Contabilidade

Financeira e Finanças pela Universidade Federal do Paraná (UFPR). Mestre em Contabilidade Financeira e Finanças pela Universidade Federal do Paraná (UFPR). Graduada em Ciências Contábeis pela Pontifícia Universidade Católica de São Paulo (PUC-SP). Docente do curso de Ciências Contábeis e Administração na Faculdade Modelo (FACIMOD). E-mail: paula.pontes.campos@gmail.com

\section{Romualdo Douglas Colauto} Pós-Doutor em Controladoria e Contabilidade pela FEA/USP. Doutor e Mestre em Engenharia de Produção na Área de Gestão de Negócios pela UFSC (2005). E-mail: rdcolauto.ufpr@gmail.com. Identificadores (ID), Lattes: http://lattes. cnpq.br/4411504880578074; Research Gate: https://www.researchgate.net/profile/ Romualdo_Colauto; Google Citations: https:// scholar.google.com/citations?user $=y 7 Z \mathrm{~m}$ GWCAAAAJ\&hl=pt-BR; Orcid: 0000-00033589-9389. 


\section{INTRODUÇÃO}

As classificações de rating de crédito são utilizadas pelos agentes financeiros desde o século XVIII (Cantor \& Packer, 1994), por proporcionarem maior transparência e auxiliar os participantes do mercado na tomada de decisão. As classificações de ratings são utilizadas com frequência pelos agentes do mercado financeiro por refletirem a probabilidade de inadimplência da entidade (Kang \& Liu, 2007); englobarem informações não acessíveis aos agentes de mercado; e sintetizarem a qualidade de crédito de um emissor ou emissão em uma escala específica (Freitas \& Minardi, 2013), auxiliando assim, na tomada de decisão dos investimentos e na precificação da dívida (Freitas \& Minardi, 2013).

Apesar dos investidores utilizarem as classificações de ratings nas decisões de investimento, os ratings não representam recomendações para que esses comprem, vendam ou mantenham seus investimentos ou ainda, medidas de valor dos ativos. Os ratings correspondem a uma avaliação da qualidade de crédito da entidade ou da probabilidade de default. Por considerarem que fatores externos não previstos no momento da atribuição das classificações possam ocorrer, as notas de rating não exprimem com exatidão a qualidade do crédito das entidades, mas fornecem probabilidades do cumprimento das obrigações no prazo determinado (Standard \& Poor's, 2014).

Os ratings de crédito apresentam implicações significativas para as entidades, pois sinalizam sua situação econômico-financeira, influenciam o custo dos empréstimos e repercutem de forma imediata em suas ações e avaliações de títulos (Jung, Soderstrom, \& Yang, 2013). As classificações de ratings também permitem o acesso a novos mercados, elevam a transparência da entidade e auxiliam os investidores, avaliadores e reguladores no processo de tomada de decisão (Standard \& Poor's, 2014).

Por apresentarem implicações significativas às entidades e considerando o fato de que as agências de risco utilizam as informações contábeis para emissão das notas de rating, por vezes os gestores são motivados a gerenciar resultados com o objetivo de obter classificações de rating mais favoráveis (Demirtas \& Cornaggia, 2013). As entidades também são motivadas a gerenciar os resultados com a finalidade de minimizar a probabilidade de default e neutralizar possíveis situações de insolvência, uma vez que as notas de rating refletem a probabilidade de inadimplência das entidades.

Considerando a contextualização exposta, a questão de pesquisa que orienta este estudo é: Qual a relação entre o gerenciamento de resultados e o rating de crédito atribuído pelas agências classificadoras de risco para companhias que negociam na $[B] 3$ ?

Apesar do gerenciamento de resultados (GR) ser objeto de estudo de diversas pesquisas (Dechow, Sloan \& Sweeney, 1995; Leuz, Nanda, \& Wysocki, 2003; Roychowdhury, 2006; Cohen \& Zarowin, 2010; Nardi \& Nakao, 2009; Liu, Ning, \& Davidson III, 2010; Ali \& Zhang, 2015), poucos o associaram às notas de rating de crédito. Dentre os estudos que abordaram esta temática, Li, Griffin, Yue e Zhao (2013) mencionaram que a maioria foi desenvolvida nos Estados Unidos, Reino Unido e Austrália, o que contribui para que o efeito sobre a economia emergente ainda não seja evidente. O principal resultado das pesquisas que abordaram este conteúdo indicou que as empresas gerenciaram seus resultados com o objetivo de alterar as classificações de rating de crédito (Ali \& Zhang, 2008; Jiang, 2008; Alissa, Bonsall, Koharki, \& Penn 2013; Demirtas \& Cornaggia, 2013; Kim, Kim, \& Song, 2013; Brown, Chen, \& Kim, 2015; Lin \& Shen, 2015).

Devido às limitações para obter os históricos de rating das entidades, poucos estudos associaram o GR e as classificações de ratings no Brasil. Apesar do estudo de Martinez e Castro (2009) verificar o efeito do income smoothing na estrutura de capital e no custo de capital de terceiros, os autores analisaram adicionalmente os ratings em ofertas públicas de debêntures e constataram que as empresas com melhores ratings apresentam maiores índices de income smoothing. Tonin e Colauto (2015) também analisaram a associação entre os ratings e o income smoothing para empresas listadas na BM\&FBovespa no período de 2005 a 2011. No entanto devido as limitações de informações, utilizaram apenas as notas de rating publicadas no exercício de 2011, não considerando uma série histórica. Os resultados sugeriram que as empresas que suavizaram resultados apresentaram maior possibilidade de receberem notas de rating.

Assim, este estudo contribui com a literatura, ao analisar uma série histórica de ratings e ao considerar as classificações de rating de crédito das três principais agências de risco (Standard \& Poor's, Fitch Ratings e Moody's), tendo em vista que a maioria dos trabalhos analisados adotou apenas uma agência de rating. Este estudo também apresenta particularidades, pois não analisa a manipulação dos dados considerando a modalidade income smoothing, mas adota modelo com accruals discricionários, tendo em vista que esses contemplam um conjunto possível de ações discricionárias (Watts \& Zimmerman, 1990).

Na perspectiva empresarial as classificações de ratings foram adotadas amplamente pelos mercados financeiros, pois os níveis e as mudanças nas classificações são capazes de prever a probabilidade de inadimplência das entidades, além de atuarem como função de governança corporativa (Kang \& Liu, 2007). Além de ser considerado como a segunda condição mais importante na tomada de decisão sobre a política financeira da empresa, pelos diretores financeiros, tendo em vista que a primeira condição é a manutenção da flexibilidade financeira (Graham \& Harvey, 2001). Desta forma, verificar a influência dos ratings no GR é relevante, uma vez que as empresas podem gerenciar seus resultados por se afastarem das classificações esperadas (Alissa et al., 2013).

Nesse contexto, é importante verificar se as empresas brasileiras gerenciam os resultados com a intenção subjacente de conturbar as percepções das agências classificadoras de riscos, tendo em vista que as informações contábeis divulgadas pelas companhias afetam diretamente a distribuição de riquezas entre acionistas; a percepção do risco (Beaver, 1981); as análises do mercado; e a alocação de recursos na economia. 


\section{GERENCIAMENTO DE RESULTADOS E RATINGS DE CRÉDITO}

O gerenciamento de resultados pode ser caracterizado como um conjunto de práticas contábeis adotadas para alterar o desempenho econômico e financeiro da entidade, com o objetivo de alterar a percepção dos agentes econômicos (Healy \& Wahlen, 1999; Scott, 2012). As alterações cujos propósitos sejam atender ao escopo dos gestores ocorrem por meio da escolha de diferentes critérios de mensuração, reconhecimento e evidenciação das informações contábeis. $\mathrm{O}$ gerenciamento de resultados é caracterizado como uma alteração intencional no processo de elaboração dos relatórios financeiros com a finalidade de obter ganhos próprios (Schipper, 1989).

Nesse contexto, os principais fatores para o gerenciamento de resultados compreendem a utilização dos accruals, utilizado nesta pesquisa como proxy para cálculo do gerenciamento; mudanças nos métodos contábeis; e alterações na estrutura de capital da entidade (Jones, 1991; Dechow et al. 1995). Os accruals proporcionam uma medida agregada das escolhas contábeis, pois ao mensurar o gerenciamento de resultados consideram o conjunto de ações discricionárias possíveis (Watts \& Zimmerman, 1990). Os accruals representam a diferença entre o lucro líquido e o fluxo de caixa operacional (Defond \& Jiambalvo, 1994), sendo segregados em accruals discricionários e não discricionários (Teoh, Welch \& Wong, 1998). Wu (2014) afirma que os Accruals Discricionários têm sido amplamente associados pela literatura ao gerenciamento de resultados, por resultarem da discricionariedade dos gestores. Enquanto os Accruals não Discricionários são caracterizados pelos lançamentos contábeis inerentes as atividades empresariais.

Nessa linha argumentativa, estudos (Jiang, 2008; Jorion, Shi \& Zhanf, 2009; Alissa et al., 2013; Demirtas \& Cornaggia, 2013; Kim, Kim \& Song, 2013; Brown, Chen \& Kim, 2015; Lin \& Shen, 2015; Miiller \& Martinez, 2016) relacionaram as práticas de gerenciamento de resultados e os ratings com a finalidade de encontrar evidências de que as empresas manipularam suas informações para influenciar as classificações atribuídas pelas agências de risco.

Alissa et al. (2013) indicaram que as empresas classificadas abaixo ou acima do esperado, gerenciaram os resultados com a finalidade de alterar as notas futuras de rating de crédito. Além disso, as empresas que apresentaram rating esperado no grau de investimento possuíram mais incentivos para gerenciar os resultados. Demirtas e Cornaggia (2013) constataram que as empresas realizaram escolhas contábeis com a finalidade de gerenciar os resultados para cima antes da classificação inicial do rating emitido pela agência Moody’s. Ao elevar os lucros, os gestores esperavam obter classificações mais favoráveis, que consequentemente reduziriam o custo da dívida. Observaram também que os aumentos das acumulações contábeis que antecederam as classificações iniciais foram revertidos nos anos seguintes.

Kim, Kim e Song (2013) detectaram o aumento do gerenciamento por meio das atividades reais em períodos que precederam as alterações de rating. Enquanto o gerenciamento de resultados por accruals reduziu significativamente, tendo em vista que as agências de risco o consideraram como um sinal negativo. Concluíram que os gestores que almejaram mudanças de rating no ano subsequente adotaram de forma mais intensa as práticas de gerenciamento por meio das atividades reais, ao invés da manipulação por accruals. Brown, Chen e Kim (2015) constataram que as empresas industriais com classificações de rating emitidas pela Standard \& Poor's (S\&P) mais próximas ao grau especulativo (BBB e BB), realizaram escolhas mais agressivas de gerenciamento de resultados. Verificaram também, que o gerenciamento de resultado não interferiu de forma negativa nas decisões de rating do ano subsequente; e que as classificações das agências de rating não foram afetadas pelo gerenciamento de resultados.

Lin e Shen (2015) verificaram que as empresas familiares presentes na base de dados do Taiwan Economic Journal, que controlaram o risco idiossincrático e adotaram práticas de gerenciamento de resultados por meio de atividades reais aumentaram as classificações de rating de crédito. Todavia, quando as empresas gerenciaram os resultados por meio dos accruals, o risco de crédito foi elevado. Miiller e Martinez (2016) investigaram se o rating de crédito na emissão de debênture foi determinado pela diferença entre o lucro contábil e tributário ou por meio do gerenciamento de resultados. Constataram que a diferença entre o lucro contábil e tributário das empresas brasileiras não determinaram o rating de crédito. Todavia, as empresas que gerenciaram os resultados tiveram seus ratings rebaixados, tendo em vista que os analistas interpretaram esse procedimento como um fator de risco de solvência.

Apesar dos estudos mencionados abordarem o risco de crédito como incentivo para o gerenciamento de resultados, alguns estudos consideraram que os ratings de crédito inibem o gerenciamento de resultados. Dentre as justificativas, esses estudos mencionam que as agências de rating podem detectar as práticas de manipulação das informações contábeis e como consequência reduzir as classificações de rating das entidades. Os estudos também indicaram que as manipulações por meio dos accruals são detectadas com maior frequência pelas agências de rating, se comparadas às manipulações das atividades reais da entidade (Kim, Kim \& Song, 2013; Lin \& Shen, 2015; Gounopoulos \& Pham, 2016).

Por certo, igualmente importante, considerar os estudos de Dechow et al. (1995) que apontam a correlação do gerenciamento de resultados (accruals discricionários) com o desempenho econômico e financeiro da empresa, conduzindo portanto a um viés no resultado da pesquisa. Uma das abordagens possíveis para controlar diretamente esses erros de especificação no modelo, é a inclusão de uma variável de controle ou outra forma de variância que controla o desempenho da empresa. Ainda relacionado ao desempenho da empresa, Adams et al. (2003) relatam que a análise da lucratividade pelos analistas financeiros possibilita examinar a capacidade da empresa em investir os superávits anuais de forma eficiente, a fim de gerar novos negócios. Ademais, os índices de lucratividade fornecem insights sobre a capacidade da administração em controlar com eficácia as despesas e estabelecer taxas competitivas de prêmio (Murcia et al. 2014). 
As pesquisas evidenciadas anteriormente indicaram que as empresas gerenciaram os resultados para obter melhores classificações de crédito. Nesse sentido, esse estudo se fundamenta no pressuposto de que as empresas gerenciam os resultados com o objetivo de interferir nas classificações de crédito, de forma a manter ou elevar as notas de rating, reduzindo assim, a probabilidade de inadimplência estimada pela agência de risco. Ao considerar a importância atribuída pelo mercado de capitais às notas de rating e as consequências dessas classificações, as entidades são motivadas a gerenciar seus resultados contábeis, com propósito de obterem melhores classificações de rating de crédito (Gray, Mirkovic \& Ragunathan, 2006; Miiller \& Martinez, 2016). Nesse contexto, apresenta-se a seguinte hipótese de teste:

$H_{(1)}:$ Há relação significativa e positiva entre os niveis de gerenciamento de resultados e as maiores notas de rating de crédito atribuidas pelas principais agências de risco.

\section{PROCEDIMENTOS METODOLÓGICOS}

O presente estudo caracteriza-se como uma pesquisa descritiva quanto ao seu objetivo, pois analisa a relação entre o gerenciamento de resultados e o rating de crédito atribuídos pelas agências classificadoras de risco para companhias que negociaram na [B] ${ }^{3}$ Brasil, Bolsa e Balcão, no período de 2010 a 2015, sendo este período selecionado em função do grau de investimento concedido ao Brasil pela Fitch, Moody's e Standard \& Poor's em setembro de 2009 até fevereiro de 2016. Período este em que o Brasil permaneceu classificado pelas agências na categoria de grau de investimento.

A população da pesquisa contempla as companhias brasileiras de capital aberto listadas na $[\mathrm{B}]^{3}$ em 31 de dezembro de 2015. Devido à ausência de dados para algumas empresas da amostra, durante o período de 2010 a 2015, as amostras consideradas integram um Painel Desbalanceado. A amostra final de rating foi composta por 59 empresas. As empresas financeiras foram excluídas, por apresentarem diversas particularidades nos procedimentos contábeis, os quais podem interferir nas proxies de mensuração do gerenciamento de resultados. Na sequência foram excluídas as empresas com ausência do rating delimitado por esta pesquisa. A delimitação se fez necessária, pois as empresas apresentam diferentes classificações de rating de crédito, que não são o foco desta pesquisa. Neste caso, consideraram-se apenas as empresas com classificações de longo prazo e em moeda estrangeira; e que fossem denominadas de emissões de longo prazo da S\&P; ratings internacionais de crédito de emissor de longo prazo da Fitch Ratings; rating emissor e rating de longo prazo da Moody's.

Os ratings de crédito são atribuídos pelas agências de risco conforme escalas específicas compostas por combinações de letras, algarismos e sinais. Normalmente, os ratings expressos por meio de letras comunicam as opiniões das agências sobre o nível relativo de risco de inadimplência. As classificações de crédito foram coletadas na base de dados Bloombergß As notas de rating de crédito foram segregadas em duas categorias, sendo: grau de investimento e grau especulativo. As empresas classificadas no grau de investimento apresentam menores riscos de crédito, por isso a probabilidade de inadimplência varia de baixa a moderada. A categoria grau especulativo, indica que as empresas possuem maior probabilidade de inadimplência, podendo em alguns casos indicar que essa inadimplência já ocorreu (Fitch Ratings, 2016).

No que diz respeito às classificações de rating, foram consideradas as notas atribuídas em escala global e em moeda estrangeira, tendo em vista que estas medem a probabilidade de pagamento na moeda da jurisdição do domicílio do emissor, considerando os riscos de transferência e conversibilidade (Fitch Ratings, 2016). Tendo em vista que as notas atribuídas pelas agências de rating são equivalentes, consideraram-se as classificações das três principais agências de rating, a fim de não limitar a amostra. As notas de rating consideradas para seleção das empresas foram representadas pelos ratings de emissão de longo prazo da S\&P; ratings internacionais de crédito de emissor de longo prazo da Fitch Ratings; rating emissor e rating de longo prazo da Moody's. Assim, para as empresas que apresentaram apenas uma dessas classificações, foram coletados os ratings históricos correspondentes. Todavia, para as empresas que apresentaram rating de mais de uma agência consideraram-se, por prudência, as piores classificações (Miiller \& Martinez, 2016).

$\mathrm{O}$ fato de as notas de rating serem expressas por meio de letras demanda sua conversão em valores numéricos, para que a operacionalização dos testes estatísticos seja possível. Neste caso, empregou-se dummies para distinguir o grau de classificação das empresas, sendo atribuído 1 para o grau de investimento e 0 para o grau especulativo. A opção por dummies decorre do fato de que o tamanho limitado da amostra inviabilizou o estudo considerando variáveis em uma escala ordinal de 1 a 8 , visto que a amostra ficou agrupada no limiar grau de investimento e grau especulativo, representados na escala ordinal por 5 e 6 . Portanto, optou-se por utilizar o modelo logit binário ao invés do probit ordinal.

Para mensuração de gerenciamento de resultado utilizou-se como proxy os Accruals Discricionários (DA), mensurados por meio da diferença entre os Accruals Totais (TA) e os Accruals não Discricionários (NDA). Os Accruals Totais foram estimados por meio da abordagem das Demonstrações dos Fluxos de Caixa, sendo obtidos pela da diferença entre o Lucro Líquido e o Fluxo de Caixa Operacional, conforme Equação 1. Para mensurar os Accruals Não Discricionários adotou-se o modelo Kothari, Leone e Wasley (2005) que utilizam o Modelo Jones Modificado (1995), mas incluem um intercepto e a variável retorno sobre ativo (ROA), corrente ou defasado, com a finalidade de especificar a performance da entidade e assim reduzir problemas de heterocedasticidade, como evidenciado na Equação 2. Dessa forma, para obtenção dos Accruals Não Discricionários, faz-se necessário primeiramente, estimar os coeficientes (Equação 3). Por fim, a partir da Equação 4, obtém-se os accruals discricionários, utilizados como Proxy para o gerenciamento de resultados. 
Em que,

$$
\begin{gathered}
T A_{i, t}=\frac{L L_{i, t}}{A_{i, t-1}}-\frac{F C O_{i, t}}{A_{i, t-1}} \\
N D A_{i, t}=\alpha_{1}+\beta_{1}\left(\frac{1}{A_{i, t-1}}\right)+\beta_{2}\left(\frac{\Delta \mathrm{R}_{\mathrm{i}, \mathrm{t}}-\Delta \mathrm{CR}_{\mathrm{i}, \mathrm{t}}}{\mathrm{A}_{\mathrm{i}, \mathrm{t}-1}}\right)+\beta_{3}\left(\frac{\mathrm{PPE}_{i, t}}{A_{i, t-1}}\right)+\beta_{4}\left(\mathrm{ROA}_{\mathrm{i}, \mathrm{t}-1}\right) \\
T A_{i, t}=\hat{\mathrm{a}}_{1}+\widehat{\beta_{1}}\left(\frac{1}{A_{i, t-1}}\right)+\widehat{\beta_{2}}\left(\frac{\Delta \mathrm{R}_{\mathrm{i}, \mathrm{t}}-\Delta \mathrm{CR}_{\mathrm{i}, \mathrm{t}}}{\mathrm{A}_{\mathrm{i}, \mathrm{t}-1}}\right)+\widehat{\beta_{3}}\left(\frac{\mathrm{PPE}_{i, t}}{A_{i, t-1}}\right)+\widehat{\beta_{4}}\left(\mathrm{ROA}_{\mathrm{i}, \mathrm{t}-1}\right)+\varepsilon_{\mathrm{i}, \mathrm{t}} \\
D A_{i, t}=T A_{i, t}-N D A_{i, t}
\end{gathered}
$$

$T A_{i, t}=$ accruals totais da empresa i no período $t$;

$N D A_{i, t}=$ accruals não discricionários da empresa $i$ no período $t$;

$D A_{i, t}=$ accruals discricionários da empresa $i$ no período $t$,

$L L_{i, t}=$ lucro líquido da empresa $i$ no final do período $t$, ponderados pelos ativos totais no final do período $t-1$;

$F C O_{i, t}=$ fluxo de caixa operacional da empresa $i$ no final do período $t$, ponderados pelos ativos totais no final do período $t-1$;

$A_{i, t-1}=$ ativos totais da empresa $i$ no final do período $t-1$.

$\Delta R_{i, t}=$ variação das receitas líquidas da empresa i do período $t-1$ para o período $t$, ponderada pelos ativos totais no final do período $t-1$;

$\Delta \mathrm{CR}_{i, t}=$ variação das contas a receber da empresa $i$ do período $t-1$ para o período $t$, ponderada pelos ativos totais no final do período $t-1$;

$P P E_{i, t}=$ saldos das contas do ativo imobilizado (bruto) e intangível da empresa $i$ no final do período $t$, ponderados pelos ativos totais

no final do período $t-1$;

$R O A_{i, t-1}=$ retorno sobre o ativo (lucro líquido/total de ativos) da empresa $i$ do período $t-1$;

$\hat{\mathrm{a}}_{1}, \beta_{1}, \beta_{2}, \beta_{3} \beta_{4}=$ coeficientes estimados; e

$\varepsilon_{\mathrm{i}, \mathrm{t}}=$ termo-de erro (resíduos) da empresa $i$ do período $t$.

A variável dependente foi composta pelas notas de rating de crédito, mensurada por meio de dummies. Devido a essa particularidade, utilizou-se o Modelo Logit Binário, como técnica de análise de dados. Os Modelos para dados binários são aplicados quando a variável dependente assume dois valores distintos, com o objetivo de prever a probabilidade de sucesso ou insucesso, condicional em um conjunto de variáveis independentes. Wooldridge (2010) menciona que a regressão logística investiga a relação entre variáveis explicativas e uma variável dependente categórica binária, neste caso, foi utilizado 1 para as empresas que apresentaram Grau de Investimento e 0 para empresas com Grau Especulativo, pressupondo inexistência de homogeneidade de variância e normalidade dos resíduos.

Ao empregar o modelo, busca-se verificar se o gerenciamento de resultados pode ser considerado fator explicativo para as notas atribuídas pelas as agências de rating. Avalia-se também, os efeitos do gerenciamento de resultados e das variáveis de controle em relação às classificações de rating de crédito. Neste estudo, o modelo é apresentado na Equação 5.

$$
L_{i, t}=\ln \left(\frac{P_{i, t}}{1-P_{i, t}}\right)=\beta_{0}+\beta_{1} D A_{i, t}+\beta_{2} T A M_{i, t}+\beta_{3} A L A V_{i, t}+\beta_{1} \operatorname{CRESC}_{i, t}+\beta_{5} \operatorname{SETOR}_{i, t}+\varepsilon_{i, t}
$$

Em que,

$\mathrm{L}_{\mathrm{t}-1}=$ variável dependente rating;

$\ln \left(\frac{P_{i, t}}{1-P_{i, t}}\right)=$ sendo que $\mathrm{P}_{\mathrm{i}, \mathrm{t}}=1$ se as empresas apresentarem grau de investimento e $1-\mathrm{P}_{\mathrm{i}, \mathrm{t}}=0$ se as empresas apresentarem grau especulativo;

$\mathrm{ADi}, \mathrm{t}=$ accruals discricionários da empresa i no período $\mathrm{t}$;

TAMi, $\mathrm{t}=$ tamanho da empresa i no período $\mathrm{t}$;

ALAVi, $\mathrm{t}=$ alavancagem da empresa i no período $\mathrm{t}$;

CRESCi,t $=$ crescimento da empresa i no período $t$;

SETORi,t $=$ setor da empresa i no período $\mathrm{t}$;

$\beta_{0}=$ intercepto do modelo;

$\beta_{1}, \beta_{2}, \beta_{3}, \beta_{4}, \beta_{5}=$ coeficientes angulares para cada variável independente.

$\varepsilon_{\mathrm{i}, \mathrm{t}}=$ erro da regressão da empresa i no período t.

As variáveis independentes, adotadas nesta pesquisa, auxiliam a análise da relação entre o gerenciamento de resultados e os ratings de crédito. As variáveis tamanho da empresa (TAM), alavancagem (ALAV), crescimento de vendas (CRESC) e setor também apresentam potencial explicativo para a relação previamente estabelecida.

A variável tamanho (TAM), mensurada por meio do logaritmo natural do ativo, é uma proxy utilizada para se referir ao porte da empresa (Poon \& Chan, 2008). Damasceno, Artes e Minardi (2008), Murcia et al. (2014), Demirtas e Cornaggia (2013), Fernandino, Takamatsu e Lamounier (2014), Pereira e Martins (2015) e Rogers, Mendes-da-Silva e Rogers (2016) constataram que empresas de maior porte possuem classificações de rating de crédito mais elevadas. Espera-se, portanto, que o tamanho das empresas esteja positivamente relacionado às notas de ratings. 
A alavancagem (ALAV) é mensurada por meio da soma do Passivo Circulante com o Passivo Não Circulante, dividido pelo Ativo Total. A partir dos resultados é possível comparar as empresas e inferir quais empresas apresentam maiores quantidades de dívidas, por meio do índice obtido. Demirtas e Cornaggia (2013) constataram que as empresas com elevada probabilidade de falência da entidade apresentaram menores notas de rating de crédito. Nesse sentido, a alavancagem está negativamente relacionada com as mudanças de rating (Kim, Kim \& Song, 2013; Murcia et al., 2014; Pereira \& Martins, 2015). Assim, espera-se encontrar uma relação negativa entre a alavancagem e as notas de rating.

No que diz respeito a variável crescimento das vendas (CRESC), Demirtas e Cornaggia (2013) constataram que as empresas com desempenho superior apresentaram maiores classificações de rating. Adams, Burton e Hardwick (2003) e Murcia et al. (2014) também encontraram evidências de que as empresas com maiores taxas de crescimento, possuem melhores notas de rating. Desse modo, espera-se encontrar uma relação positiva entre o crescimento das vendas e as notas de rating. A variável crescimento foi mensurada da seguinte forma: receita líquida $t$ - receita líquida de $t-1$. O resultado obtido foi dividido pela receita líquida de $\mathrm{t}-1$.

A variável setor é operacionalizada por meio de dummies, sendo atribuído (1) para as empresas que se enquadravam no respectivo setor ou (0) para as empresas que não eram pertentes aquele setor. Devido as empresas que compõem a amostra, a variável setor foi segmentada em 8 variáveis dummies, a saber: bens industriais; consumo cíclico; consumo não cíclico; materiais básicos; petróleo, gás e biocombustíveis; saúde; telecomunicações; e utilidade pública. Tendo em vista que alguns setores apresentam maiores riscos que outros, Damasceno, Artes e Minardi (2008) afirma que os setores são importantes no momento da atribuição das notas de rating. Apesar da importância mencionada por Damasceno, Artes e Minardi (2008), não foi possível estimar uma regressão para cada setor devido as limitações associadas a amostra, visto que o setor saúde apresentou 1 empresa, enquanto o de utilidade pública estava 20 empresas.

A escolha da regressão logística binária ao invés do modelo logístico multinomial decorre em função do reduzido número de categorias da amostra (Fernandino, Takamatsu \& Lamounier, 2014). Os autores ainda mencionaram que o emprego de diversas variáveis dependentes reduziria os graus de liberdade, influenciando a capacidade de análise da significância estatística dos parâmetros da equação 5 .

Para estimar os coeficientes do modelo regressivo de Kothari, Leone e Wasley (2005), referente ao gerenciamento de resultados, utilizou-se o modelo regressivo com dados em painel. Os testes estatísticos e as análises das regressões foram realizados com por meio do software $\operatorname{Stata}^{\circledR} 13$.

\section{DESCRIÇÃO E ANÁLISE DE RESULTADOS}

Os accruals discricionários utilizados como proxy do gerenciamento de resultados foram mensurados por meio do modelo de Kothari, Leone e Wasley (2005). Os resultados dos testes estatísticos do modelo Kothari, Leone e Wasley (2005) compreenderam 354 observações referente ao período de 2010 a 2015, como apresentado na Tabela 1.

Tabela 1 - Estimativas dos accruals discricionários pelo modelo Kothari et al. (2005) para amostra Rating

\begin{tabular}{|c|c|c|c|c|}
\hline$R^{2}$ within & 0,9251 & & $\begin{array}{l}\text { Número de } \\
\text { observações }\end{array}$ & 350 \\
\hline $\begin{array}{c}\text { between } \\
\text { overall }\end{array}$ & $\begin{array}{l}0,3411 \\
0,8108\end{array}$ & & $\begin{array}{l}\text { Número de grupos } \\
\qquad \begin{array}{c}F(4,58) \\
\text { Prob }>F\end{array}\end{array}$ & $\begin{array}{c}59 \\
11832,57 \\
0,0000\end{array}$ \\
\hline Variáveis & Coeficiente & $\begin{array}{l}\text { Erro padrão } \\
\text { robusto }\end{array}$ & $\mathbf{T}$ & $P>|t|$ \\
\hline Constante & $-0,043$ & 0,007 & $-5,98$ & $0,000 * \star \star$ \\
\hline $1 / \mathrm{A}_{\mathrm{i}, \mathrm{t}-1}$ & 25,614 & 20,324 & 1,26 & 0,213 \\
\hline$\Delta \mathrm{R}-\Delta \mathrm{CR} / \mathrm{A}_{\mathrm{i}, \mathrm{t}-1}$ & $-0,031$ & 0,017 & $-1,78$ & $0,080^{*}$ \\
\hline $\mathrm{PPE} / \mathrm{A}_{\mathrm{i}, \mathrm{t}-1}$ & $-0,070$ & 0,009 & $-7,73$ & $0,000 * \star \star$ \\
\hline $\mathrm{ROA}_{\mathrm{t}-1}$ & 1,166 & 0,074 & 15,75 & $0,000 * \star \star *$ \\
\hline
\end{tabular}

Nota: Variável dependente accruals totais (TA), obtida pelo enfoque dos Fluxos de Caixa: Lucro líquido - fluxo de caixa operacional, ponderadas pelo ativo deflacionado.

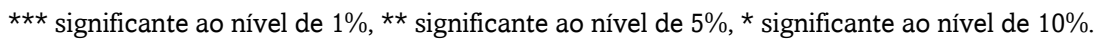

Fonte: Dados da pesquisa.

Ao estimar os accruals discricionários verificou-se normalidade dos dados. Apesar dos mesmos não seguirem uma distribuição normal, Wooldridge (2010) fundamentando-se no Teorema do Limite Central, discorre que os estimadores 
de Mínimos Quadrados Ordinários (MQO) satisfazem a normalidade assintótica, indicando que eles aproximadamente possuem distribuição normal em amostras suficientemente grandes.

Ao realizar o teste de Durbin-Watson, os resultados da estatística d $(1,1389)$ indicaram problemas de autocorrelação. Os problemas de autocorrelação ocorrem quando os resíduos incorporam os efeitos de uma variável excluída do modelo. Analisando os problemas de heterocedasticidade, os resultados do Teste Breusch-Pagan / Cook-Weisberg ( $p$-valor 0,000 ) rejeitaram a hipótese nula que os dados não apresentam heterocedasticidade. Neste caso, como os resultados dos testes indicaram violação dos pressupostos de uma regressão linear, medidas corretivas foram adotadas.

O diagnóstico de multicolinearidade foi realizado por meio do teste de Fatores de Inflacionamento da Variância (VIF). Tendo em vista que os valores estimados se aproximam de 1, o modelo não apresentou problemas de multicolinearidade. Na Tabela 1, o valor do $\mathrm{R}^{2}$ within estimado por efeitos fixos indica que o modelo explica $92,51 \%$ da variação do gerenciamento de resultados dentro das empresas da amostra, ou seja, o quanto as variáveis explicativas do modelo justificam as alterações do gerenciamento de resultados dentro de cada uma das empresas ao longo do tempo. Ao passo que o valor indicado do $\mathrm{R}^{2}$ between significa que o modelo explica $34,11 \%$ da variação do gerenciamento de resultados entre as empresas da amostra, ou seja, o quanto as variáveis explicativas capturam as diferenças no gerenciamento de resultados entre as empresas da amostra. Por fim, o $\mathrm{R}^{2}$ overall reflete a média ponderada de $81,08 \%$ entre o $\mathrm{R}^{2}$ within e $\mathrm{R}^{2}$ between.

Os resultados estatísticos indicam que as variáveis PPE/A $\mathrm{i}_{\mathrm{i}, \mathrm{-}-1}$ (Propriedade, Plantas e Equipamentos) e ROA $\mathrm{A}_{\mathrm{t}-1}(\mathrm{Retorno}$ sobre o Ativo), possuem valor preditivo significante de $1 \%$ para explicar o comportamento dos accruals totais. Enquanto a variável $\Delta \mathrm{R}-\triangle \mathrm{CR} / \mathrm{A}_{\mathrm{i}, \mathrm{t}-1}$ (variação da receita líquida - variação de contas a receber) apresentou significância ao nível de $10 \%$. O sinal negativo do coeficiente da variável PPE/ $\mathrm{A}_{\mathrm{i}, \mathrm{t}-1}$ está de acordo com o comportamento retratado em Jones (1991), uma vez que esse está associado aos accruals redutores do lucro como depreciação e amortização. O sinal positivo do ROA também está de acordo com o relatado por Kothari, Leone e Wasley (2005), indicando que a performance da entidade contribui para explicação dos accruals. Os resultados sugerem que as empresas da amostra gerenciam seus resultados. Apesar da variável $1 /$ $\mathrm{A}_{\mathrm{i}, \mathrm{t}-1}$ não apresentar significância estatística, os coeficientes foram mantidos, pois a intenção não é validar o modelo de Kothari, Leone e Wasley (2005), uma vez que esse se encontra estabelecido na literatura para fins de previsão.

As notas de rating representam a qualidade de crédito das entidades por meio de classificações qualitativas, ou seja, as notas são compostas por letras, sinais e números. Em virtude dessa particularidade, as pesquisas que envolvem classificações de rating conferem valores a essas notas, por meio de dummy ou escala ordinal, tornando possível a operacionalização dos testes estatísticos. Este estudo atribuiu valores as notas de rating por meio de dummies, destinando 1 para as empresas com classificações no Grau Investimento e 0 para as empresas com Grau Especulativo. A Tabela 2 expõe, anualmente, a frequência das notas de rating relacionadas ao grau de investimento.

Tabela 2 - Frequência das notas de ratings do Grau de Investimento

\begin{tabular}{|c|c|c|c|c|c|c|c|c|c|c|c|c|}
\hline \multirow{2}{*}{$\begin{array}{c}\text { Notas de } \\
\text { Rating }\end{array}$} & \multicolumn{2}{|c|}{2010} & \multicolumn{2}{|c|}{2011} & \multicolumn{2}{|c|}{2012} & \multicolumn{2}{|c|}{2013} & \multicolumn{2}{|c|}{2014} & \multicolumn{2}{|c|}{2015} \\
\hline & Freq. & $\%$ & Freq. & $\%$ & Freq. & $\%$ & Freq. & $\%$ & Freq. & $\%$ & Freq. & $\%$ \\
\hline AAA/Aaa & & & & & & & & & & & & \\
\hline $\mathrm{AA}+/ \mathrm{Aa} 1$ & & & & & & & & & & & & \\
\hline $\mathrm{AA} / \mathrm{Aa} 2$ & & & & & & & & & & & & \\
\hline $\mathrm{AA}-/ \mathrm{Aa} 3$ & & & & & & & & & & & & \\
\hline $\mathrm{A}+/ \mathrm{A} 1$ & & & & & & & & & & & & \\
\hline A / A2 & & & & & 1 & 1,7 & 1 & 1,85 & 1 & 1,85 & & \\
\hline A- / A3 & & & 2 & 3,39 & 1 & 1,7 & 1 & 1,85 & 1 & 1,85 & & \\
\hline $\mathrm{BBB}+/ \mathrm{Baa} 1$ & 2 & 3,39 & 1 & 1,69 & 1 & 1,7 & 1 & 1,85 & 1 & 1,85 & & \\
\hline BBB / Baa2 & 1 & 1,69 & 4 & 6,78 & 6 & 10,17 & 7 & 12,96 & 6 & 11,11 & & \\
\hline BBB- / Baa3 & 11 & 18,64 & 14 & 23,73 & 11 & 18,64 & 11 & 20,37 & 15 & 27,78 & & \\
\hline Total & 14 & 23,72 & 21 & 35,59 & 20 & 33,91 & 21 & 38,88 & 24 & 44,44 & 0 & 0 \\
\hline
\end{tabular}

Fonte: Dados da pesquisa.

Ao analisar a Tabela 2 observa-se que as agências de risco não atribuíram a nenhuma empresa da amostra notas que correspondessem a qualidade máxima de crédito, representada pelas notas AAA/Aaa. No entanto, observa-se um predomínio de empresas com classificações BBB-/Baa3, indicando que tais empresas podem ser rebaixadas a categoria de grau especulativo, devido ao sinal negativo. Os estudos de Brown et al. (2015) apontaram que empresas do setor industrial com classificações de rating próximas ao grau especulativo BBB e BB, optaram por incentivos mais agressivos no gerenciamento de resultados. 
Ao analisar o ano de 2015 observou-se o rebaixamento das notas de rating da categoria grau de investimento para grau especulativo. Essas reduções são reflexos de fatores endógenos e exógenos que acabam interferindo nas avaliações das agências de risco. Possivelmente, as notas de rating foram alteradas em função do rebaixamento do risco soberano brasileiro e das dificuldades econômicas que o país vem enfrentando, e que acabam interferindo no desenvolvimento da empresa.

A Tabela 3 apresenta a frequência das notas de rating relacionadas ao grau especulativo.

Tabela 3 - Frequência das notas de ratings do Grau Especulativo

\begin{tabular}{|c|c|c|c|c|c|c|c|c|c|c|c|c|}
\hline \multirow{2}{*}{$\begin{array}{c}\text { Notas de } \\
\text { Rating }\end{array}$} & \multicolumn{2}{|c|}{2010} & \multicolumn{2}{|c|}{2011} & \multicolumn{2}{|c|}{2012} & \multicolumn{2}{|c|}{2013} & \multicolumn{2}{|c|}{2014} & \multicolumn{2}{|c|}{2015} \\
\hline & Freq. & $\%$ & Freq. & $\%$ & Freq. & $\%$ & Freq. & $\%$ & Freq. & $\%$ & Freq. & $\%$ \\
\hline $\mathrm{BB}+/ \mathrm{Ba} 1$ & 8 & 13,56 & 10 & 16,95 & 10 & 16,95 & 12 & 22,22 & 10 & 18,52 & 1 & 1,92 \\
\hline $\mathrm{BB} / \mathrm{Ba} 2$ & 9 & 15,26 & 7 & 11,86 & 11 & 18,64 & 11 & 20,37 & 10 & 18,52 & 3 & 5,77 \\
\hline $\mathrm{BB}-/ \mathrm{Ba} 3$ & 9 & 15,26 & 8 & 13,56 & 4 & 6,78 & 2 & 3,70 & 3 & 5,56 & 11 & 21,15 \\
\hline $\mathrm{B}+/ \mathrm{B} 1$ & 1 & 1,69 & 3 & 5,09 & 2 & 3,39 & 1 & 1,85 & 3 & 5,56 & 13 & 25,0 \\
\hline $\mathrm{B} / \mathrm{B} 2$ & 3 & 5,08 & & & 2 & 3,39 & 3 & 5,56 & 2 & 3,7 & 13 & 25,0 \\
\hline B- / B3 & 2 & 3,39 & 3 & 5,09 & 1 & 1,69 & 2 & 3,71 & & & 3 & 5,77 \\
\hline $\mathrm{CCC}+$ / Caa 1 & & & & & & & & & 1 & 1,85 & 4 & 7,69 \\
\hline CCC / Caa2 & & & 1 & 1,69 & 1 & 1,69 & & & & & 1 & 1,92 \\
\hline CCC- / Caa3 & & & & & & & & & 1 & 1,85 & 2 & 3,85 \\
\hline $\mathrm{CC} / \mathrm{Ca}$ & & & & & & & & & & & & \\
\hline $\mathrm{C} / \mathrm{C}$ & & & & & & & & & & & & \\
\hline $\mathrm{D} /-$ & & & & & 2 & 3,39 & 2 & 3,71 & & & 1 & 1,93 \\
\hline Total & 32 & 54,24 & 32 & 54,24 & 33 & 55,92 & 33 & 61,12 & 30 & 55,56 & 52 & 100 \\
\hline
\end{tabular}

Fonte: Dados da pesquisa.

Ao analisar as classificações do grau especulativo, observa-se que ao longo dos 6 anos, as classificações das empresas indicaram boa qualidade de crédito para a maioria das empresas. Por outro lado, o grupo de empresas com classificações $\mathrm{B}^{+} / \mathrm{B} 1$ e B/B2 apresentaram incertezas financeiras, sendo vulneráveis ao não cumprimento de suas obrigações.

Em relação ao exercício de 2012 observa-se o rebaixamento de duas empresas ao nível default. Na comparação entre 2013 e 2014 observou-se uma melhora nas classificações das entidades, pois estas passaram a integrar o grau de investimento. A ausência de notas referente às notas $\mathrm{CC} / \mathrm{Ca}$ e $\mathrm{C}$ sugere que as agências não estão classificando as empresas com elevado risco de crédito, mas a estão classificando diretamente em default. De acordo com Kim et al. (2013), Lin e Shen (2015) e Gounopoulos e Pham (2016), isso pode ocorrer devido à verificação das agências de rating, com maior frequência, pelas manipulações por meio dos accruals que manipulações por atividades reais da empresa.

Ao realizar uma análise global (2010-2015) das classificações de rating, é possível observar que no decorrer dos 6 anos, houve uma maior concentração de empresas próximos ao limiar entre o grau de investimento e grau especulativo, como evidenciado por Silva (2012) e Murcia et al. (2014).

As estatísticas descritivas dos Accruals Discricionários, Tamanho, Alavancagem e Crescimento das Vendas são evidenciadas na Tabela 4, com o propósito de inferir sobre as principais medidas de dispersão das variáveis, que compõem a amostra Rating.

Tabela 4 - Estatística Descritiva das Variáveis da Amostra Rating

\begin{tabular}{c|c|c|c|c|c}
\hline Varáveis & Obs. & Média & Desvio Padrão & Mínimo & Máximo \\
\hline $\begin{array}{c}\text { Accruals } \\
\text { Discricionários }\end{array}$ & 351 & 0,096 & 0,173 & 0,000 & 2,001 \\
Tamanho & 351 & 9,388 & 1,405 & 3,187 & 14,753 \\
Alavancagem & 351 & 0,676 & 0,865 & 0,075 & 16,358 \\
$\begin{array}{c}\text { Crescimento das } \\
\text { Vendas }\end{array}$ & 350 & 0,269 & 2,580 & $-1,000$ & 48,030 \\
\hline
\end{tabular}

Fonte: Dados da pesquisa. 
Os accruals discricionários são evidenciados na Tabela 4 em termos absolutos, pois mensura-se a amplitude do gerenciamento e não o fato dele ser negativo ou positivo. A estatística descritiva demonstra que as empresas gerenciam os resultados para atender a objetivos específicos, tais como: não divulgar perdas anuais (Roychowdhury, 2006; Reis, Lamounier \& Bressan, 2015); atender as previsões dos analistas (Dhaliwal, Gleason \& Mills, 2004); iludir os investidores em função dos incentivos recebidos do mercado de capitais. Ao analisar dispersão, observa-se que esta não é elevada, indicando que em média as observações para esta variável permanecem próximas à zero. Em relação aos valores de gerenciamento de resultados, observa-se o valor máximo de accruals mensurados foi 2,001, indicando que as empresas gerenciaram os resultados.

O tamanho da empresa, mensurado por meio do Logaritmo Natural do Ativo Total, foi influenciado pelo ativo das companhias de maior porte, como indicado pelas medidas de amplitude e pela heterogeneidade da amostra (Murcia et al., 2014; Pereira \& Martins, 2015). Quanto à alavancagem, constatou-se que em média, as empresas da amostra optam por utilizar capital de terceiros, para financiamento de seus ativos, tendo em vista que os ativos são financiados na proporção de $32,40 \%$ do capital próprio e $67,60 \%$ do capital de terceiros, como observado nos estudo de Soares, Coutinho e Camargos (2012) e Demirtas e Cornaggia (2013) e Rogers et al. (2016). Em relação ao crescimento das vendas, observa-se que este apresenta maior dispersão quando comparados às demais variáveis da amostra Rating (Demirtas \& Cornaggia, 2013; Murcia et al., 2014). A elevada dispersão pode ser resultante das particularidades empresariais, uma vez que a amostra apresenta empresas classificadas em setores distintos.

Para verificar a relação entre o gerenciamento de resultados e os ratings de créditos atribuídos pelas agências classificadores de riscos utilizou-se o modelo Logit binário para dados em painel. Dentre as características desse modelo, destaca-se a utilização de uma variável dependente não contínua. Vale ressaltar, que o painel é desbalanceado devido à ausência de classificações de ratings e informações contábeis necessárias para todos os períodos de uma mesma empresa. Na Tabela 5 evidenciam-se os resultados obtidos por meio da aplicação do modelo logit binário para painel.

Tabela 5 - Resultado Logit para Painel

\begin{tabular}{|c|c|c|c|c|}
\hline & & \multicolumn{2}{|c|}{ Número de observações } & 311 \\
\hline \multicolumn{2}{|c|}{ Log likelihood $=-108,51318$} & \multicolumn{2}{|c|}{ Número de grupos } & 59 \\
\hline & & \multicolumn{2}{|c|}{ Wald chi2(11) } & 17,54 \\
\hline & & \multicolumn{2}{|c|}{ Prob > chi2 } & 0,0929 \\
\hline GrauInv & Coeficiente & Erro Padrão & $\mathbf{T}$ & $P>|t|$ \\
\hline Constante & $-10,724$ & 4,840 & $-2,220$ & $0,027 * \star$ \\
\hline DA & $-10,886$ & 6,189 & $-1,760$ & $0,079^{\star}$ \\
\hline Tam & 1,323 & 0,504 & 2,630 & $0,009 * \star \star$ \\
\hline Alav & $-4,134$ & 3,213 & $-1,290$ & 0,198 \\
\hline Cresc & $-0,335$ & 1,061 & $-0,320$ & 0,752 \\
\hline Bens Ind & $-2,667$ & 2,312 & $-1,150$ & 0,249 \\
\hline $\mathrm{CC}$ & $-2,554$ & 2,484 & $-1,030$ & 0,304 \\
\hline $\mathrm{CNC}$ & $-1,202$ & 1,884 & $-0,640$ & 0,524 \\
\hline Mat Bas & $-0,637$ & 1,759 & $-0,360$ & 0,717 \\
\hline PGBio & $-0,169$ & 2,669 & $-0,060$ & 0,950 \\
\hline Saúde & $-27,694$ & 13532,420 & 0,000 & 0,998 \\
\hline Telecom & 4,453 & 3,771 & 1,180 & 0,238 \\
\hline U Pub & 0,000 & (omitted) & & \\
\hline
\end{tabular}

Nota . $\mathrm{DA}=$ accruals discricionários; Tam $=$ tamanho; Alav = alavancagem $;$ Cresc $=$ crescimento; Bens Ind $=$ bens industriais; $\mathrm{CC}=$ consumo cíclico; $\mathrm{CNC}=$ consumo não cíclico; Mat Bas = materiais básicos; $\mathrm{PGBio}=$ petróleo, gás e biocombustíveis; Telecom = telecomunicações; U Pub. = utilidade pública; /cur = intercepto.

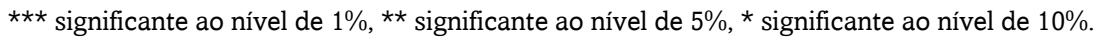

Fonte: Dados da pesquisa.

Observa-se uma relação inversa entre os accruals discricionários e os ratings de crédito, rejeitando a hipótese de que há relação positiva entre os níveis de gerenciamento e as notas de rating. Apesar do coeficiente negativo, a relação entre 
ambas se mostrou significante, ao nível de 10\%. Esses resultados contrariam os resultados de Alissa et al. (2013) e Demirtas e Cornaggia (2013), que verificaram que as empresas que gerenciaram seus resultados apresentaram notas de rating superiores. Infere-se que a redução das notas de ratings ocorra, devido a interpretação do gerenciamento de resultados pelos analistas como um fator de risco de solvência. Lin e Shen (2015) e Gounopoulos e Pham (2016) mencionam que o gerenciamento por accruals é detectado com mais facilidade pelas agências de risco e como consequência, observa-se o rebaixamento das notas de rating.

A variável tamanho mostrou-se significante ao nível de $1 \%$, confirmando que as empresas de maior porte apresentaram notas de rating superiores (Damasceno, Artes \& Minardi, 2008; Fernandino, Takamatsu \& Lamounier, 2014). Infere-se que por serem mais estáveis, são capazes de preservar a qualidade de seus créditos e como consequência obter notas superiores. Damasceno et al. (2008), Alissa et al. (2013), Demirtas e Cornaggia (2013), Fernandino, Takamatsu e Lamounier (2014), Murcia et al. (2014), Pereira e Martins (2015) e Rogers, Mendes-da-Silva e Rogers (2016) também encontram uma relação positiva entre o rating e o porte empresarial, confirmando os achados dessa pesquisa.

Apesar da alavancagem não ser significativa, o sinal negativo atendeu a relação esperada, pois empresas com altos níveis de alavancagem apresentam menores classificações de rating (Gray, Mirkovic \& Ragunathan, 2006; Rogers, Mendes-da-Silva \& Rogers, 2016), em função do não cumprimento de obrigações (Kim, Kim \& Song, 2013; Murcia et al., 2014). desta forma, as empresas com melhores classificações de rating dependem menos do capital de terceiros (Pereira \& Martins, 2015).

No que diz respeito à relação entre crescimento das vendas das entidades e o rating de crédito, o sinal negativo contradiz a relação esperada de que as empresas em crescimento recebem melhores classificações (Demirtas \& Cornaggia, 2013; Murcia et al., 2014), por apresentarem forte desempenho de fluxos de caixa e maior valor econômico (Adams, Burton \& Hardwick, 2003). O coeficiente negativo pode ser explicado pela escolha de outras fontes para financiamento de seu crescimento.

Apesar de não significantes, com exceção do setor de telecomunicações, os demais apresentaram sinal semelhante ao obtido por Rogers, Mendes-da-Silva e Rogers (2016). Quanto ao setor de utilidade pública, este foi omitido por apresentar multicolinearidade.

\section{CONCLUSÕES}

As notas de rating de crédito atuam como parâmetros da qualidade do crédito empresarial, sendo utilizadas pelos agentes econômicos para verificar o risco de crédito, definir as estruturas do financiamento, negociar os títulos empresariais e prever a probabilidade de inadimplência das entidades. Por mensurar a probabilidade de default das entidades, os ratings são relevantes para o mercado financeiro. As agências de risco consideram diversos fatores para definição e atribuição das notas de rating, como por exemplo, as informações contábeis. Neste caso, os ratings podem atuar como motivadores da prática do gerenciamento de resultados, objetivando minimizar a probabilidade de default das entidades e como consequência influenciar as classificações de rating.

As empresas podem ser motivadas a gerenciar os resultados a fim de obter melhores classificações de rating de crédito para influenciar a percepção de risco dos agentes do mercado. Nesse contexto, o presente estudo verificou a relação entre o nível de gerenciamento de resultados e riscos de créditos atribuídos pelas agências de ratings para companhias que negociam na $[\mathrm{B}]^{3}$. A hipótese que motivou o desenvolvimento deste estudo está relacionada ao fato de que as empresas podem gerenciar seus resultados com a intenção de influenciar as percepções das agências classificadoras de riscos e minimizar seu risco de crédito.

Em razão da tendência das empresas em gerenciar seus resultados para influenciar as classificações, esperava-se uma relação positiva e significativa entre os níveis de gerenciamento de resultados e as notas de rating. No entanto, os resultados revelaram uma relação inversa, apesar da significância estatística ao nível de 10\%. Analisando ainda as classificações de rating, observa-se que as empresas brasileiras apresentaram concentração de notas em torno do limiar entre o grau de investimento e grau especulativo. Neste caso, esperava-se um gerenciamento dos resultados mais intenso e agressivo.

Apesar dos resultados contrariarem as evidências teóricas existem explicações alternativas para os resultados desta pesquisa. Antes de tudo, empresas com classificações superiores, não gerenciam seus resultados com a mesma intensidade que as empresas do grau especulativo ou que podem ser rebaixadas de categoria, por apresentarem maior qualidade de crédito. Além disso, as agências de risco podem ter detectado as práticas de manipulação e ter reduzido as classificações de rating. Por fim, outros fatores são considerados na atribuição das notas de rating, e neste caso, o gerenciamento pode não ter influência a ponto de interferir nas classificações, tendo em vista que fatores exógenos também comprometem as notas de rating.

Apesar dos procedimentos realizados, esta pesquisa possui algumas limitações. No que diz respeito aos ratings, optou-se por trabalhar apenas com emissões em escala global e em moeda estrangeira, excluindo as emissões realizadas pelas agências nacionais. Em relação à categorização da variável dependente rating, recomenda-se a adoção de uma escala ordinal, o que poderia levar a outros resultados em função do modelo utilizado. Não foi possível categorizar as notas de rating em uma escala ordinal, pois as classificações se concentraram em torno do grau de investimento e grau especulativo. Por este motivo, adotou-se uma variável binária. Quanto ao gerenciamento de resultados, outros modelos presentes na literatura acadêmica poderiam melhor capturar os accruals.

De posse dos resultados dessa pesquisa, sugere-se como recomendações para estudos futuros, que a relação entre os níveis de gerenciamento de resultados e as notas de rating das empresas listadas na América Latina seja investigada, 
para verificar se os resultados obtidos se assemelham aos dessa pesquisa. Sugere-se ainda, analisar a relação entre o gerenciamento de resultados e as alterações das notas de rating (dowgrade/upgrade) para as empresas da América Latina.

\section{REFERENCIAS}

Adams, M., Burton, B., \& Hardwick, P. (2003). The determinants of credit ratings in the United Kingdom insurance industry. Journal of Business Finance \& Accounting, 30(3-4), 539-572.

Ali, A., \& Zhang, W. (2008). Proximity to broad credit rating change and earnings management. [Working Paper]. Social Science Research Network. Recuperado de: <http://ssrn.com/abstract=1163003>.

Ali, A., \& Zhang, W. (2015). CEO tenure and earnings management. Journal of Accounting and Economics, 59(1), 60-79.

Alissa, W., Bonsall, S. B., Koharki, K., \& Penn, M. W. (2013). Firms' use of accounting discretion to influence their credit ratings. Journal of Accounting and Economics, 55(2), 129-147.

Beaver, W. H. (1981). Financial reporting: an accounting revolution. Prentice Hall.

Brown, K., Chen, V. Y. S., \& Kim, M. (2015). Earnings management through real activities choices of firms near the investmentspeculative grade borderline. Journal of Accounting and Public Policy, 34(1), 74-94.

Cantor, R., \& Packer, F. (1994). The Credit Rating Industry. Quarterly Review, 19(2), 1-26.

Cohen, D. A., \& Zarowin, P. (2010). Accrual-based and real earnings management activities around seasoned equity offerings. Journal of Accounting and Economics, 50(1), 2-19.

Damasceno, D. L., Artes, R., \& Minardi, A. M. A. F. (2008). Determinação de rating de crédito de empresas brasileiras com a utilização de índices contábeis. Revista de Administração, 43(4), 344-355.

Dechow, P. M., Sloan, R. G., \& Sweeney, A. P. (1995). Detecting earnings management. Accounting review, 70(2), 193-225.

DeFond, M. L., \& Jiambalvo, J. (1994). Debt covenant violation and manipulation of accruals. Journal of accounting and economics, 17(1-2), 145-176. 135-145.

Demirtas, K. O., \& Cornaggia, K. R. (2013). Initial credit ratings and earnings management. Review of Financial Economics, 22(4),

Dhaliwal, D. S., Gleason, C. A., \& Mills, L. F. (2004). Last-chance earnings management: Using the tax expense to meet analysts' forecasts. Contemporary Accounting Research, 21(2), 431-459.

Fernandino, G. F., Takamatsu, R. T., \& Lamounier, W. M. (2014). Impacto dos Índices Contábeis na Aplicação de Rating de Crédito em Empresas Brasileiras de Capital Aberto. Contabilidade Vista \& Revista, 25(3), 78-94.

Fitch Ratings. (2016). Emissores Internacionais e Escalas Ratings de Crédito. Recuperado de: https://www.fitchratings.com.br/ pages/def_rtg_credit_emissor2?p=rtg_escala_lp_1\#rtg_escala_lp_1.

Freitas, A. D. P. N., \& Minardi, A. M. A. F. (2013). The impact of credit rating changes in Latin American stock markets. BAR-Brazilian Administration Review, 10(4), 439-461.

Gray, S., Mirkovic, A., \& Ragunathan, V. (2006). The determinants of credit ratings: Australian evidence. Australian Journal of Management, 31(2), 333-354.

Graham, J. R., \& Harvey, C. R. (2001). The theory and practice of corporate finance: Evidence from the field. Journal of financial economics, 60(2), 187-243 counting

Gounopoulos, D., \& Pham, H. (2016). Credit Ratings and Earnings Management around IPOs. Journal of Business Finance \& Ac-

Jiang, J. (2008). Beating earnings benchmarks and the cost of debt. The Accounting Review, 83(2), 377-416.

Jones, J. J. (1991). Earnings management during import relief investigations. Journal of accounting research, 193-228.

Jorion, P., Shi, C., \& Zhang, S. (2009). Tightening credit standards: the role of accounting quality. Review of Accounting Studies, 14(1), 123-160.

Jung, B., Soderstrom, N., \& Yang, Y. S. (2013). Earnings smoothing activities of firms to manage credit ratings. Contemporary Accounting Research, 30(2), 645-676.

Kang, Q., \& Liu, Q. (2007). Credit rating changes and CEO incentives. [Working Paper]. Social Science Research Network. Retrieved from http://ssrn.com/abstract=971277.

Kim, Y. S., Kim, Y., \& Song, K. (2013). Credit rating changes and earnings management. Asia-Pacific Journal of Financial Studies, 42(1), 109-140.

Kothari, S. P., Leone, A. J., \& Wasley, C. E. (2005). Performance matched discretionary accrual measures. Journal of accounting and economics, 39(1), 163-197.

Leuz, C., Nanda, D., \& Wysocki, P. (2003). Earnings Management and Investors Protection: An International Comparison. Journal of Financial Economics, 3(69), 505-527. $1-22$.

Li, K., Griffin, D., Yue, H., \& Zhao, L. (2013). How does culture influence corporate risk-taking? Journal of Corporate Finance, 23, 
Lin, Y. M., \& Shen, C. A. (2015). Family firms' credit rating, idiosyncratic risk, and earnings management. Journal of Business Research, 68(4), 872-877.

Liu, Y., Ning, Y., \& Davidson III, W. N. (2010). Earnings management surrounding new debt issues. Financial Review, 45(3), 659-681.

Martinez, A. L., \& Castro, M. A. R. (2009). Ratings, custo de capital de terceiros e alisamento de resultados no Brasil. Encontro Brasileiro de Finanças São Leopoldo, RS. Anais... do SBFin.

Miiller, D. L., \& Martinez, A. L. (2016). Book-tax difference, earnings management and bond ratings in the Brazilian market. Revista Universo Contábil, 12(3), 91

Murcia, F. C. de S., Murcia, F. D., Rover, S., \& Borba, J. A. (2014). The determinants of credit rating: Brazilian evidence. BAR-Brazilian Administration Review, 11(2), 188-209.

Nardi, P. C. C., \& Nakao, S. H. (2009). Gerenciamento de resultados e a relação com o custo da dívida das empresas brasileiras abertas. Revista Contabilidade \& Finanças, 20(51), 77-100.

Pereira, L. H. M., \& Martins, O. S. (2015). Rating de crédito, governança corporativa e desempenho das empresas listadas na BM\&FBovespa. REGE Revista de Gestão, 22(2), 205-221.

Poon, W. P., \& Chan, K. C. (2008). An empirical examination of the informational content of credit ratings in China. Journal of Business Research, 61(7), 790-797.

Reis, E. M. dos., Lamounier, W. M., \& Bressan, V. G. F. (2015). Evitar Divulgar Perdas: Um Estudo Empírico do Gerenciamento de Resultados por Meio de Decisões Operacionais. Revista Contabilidade \& Finanças. 26(69), 247-260.

Rogers, D., Mendes-da-Silva, W. \& Rogers, P. (2016). Credit rating change and capital structure in Latin America. BAR-Brazilian Administration Review. 13(2).

Roychowdhury, S. (2006). Earnings management through real activities manipulation. Journal of accounting and economics, 42(3), 335-370.

Silva, D. R. (2012) Associações entre rating de crédito e estrutura de capitais de empresas listadas na América Latina. Tese (Doutorado), Fundação Getúlio Vargas, São Paulo.

Soares, G. D. O. G., Coutinho, E. S. \& Camargos, M. A. de. (2012). Determinantes do Rating de Crédito de Companhias Brasileiras. Contabilidade Vista \& Revista, 23(3), 109-143.

Standard and Poor's. (2014). Guide to credit rating essentials: What are credit ratings and how do they work? Disponível em: http:// www.spratings.com/documents/20184/760102/SPRS_Understanding-Ratings_GRE.pdf/298e606f-ce5b-4ece-9076-66810cd9b6aa

Teoh, S. H., Welch, I. \& Wong, T. J. (1998). Earnings management and the underperformance of seasoned equity offerings. Journal of Financial Economics, 50(1), 63-99.

Tonin, J. M. F. \& Colauto, R. D. (2015). Relação entre Income Smoothing e Ratings em Companhias Brasileiras de Capital Aberto. Contabilidade Vista \& Revista, 26(1), 104-122.

Watts, R. L. \& Zimmerman, J. L. (1990). Positive accounting theory: a ten-year perspective. The Accounting Review, 65(1), $131-156$.

Wu, RS. (2014). Predicting earnings management: A nonlinear approach. International Review of Economics \& Finance, 30, 1-25.

Wooldridge, J. M. 2010. Econometric analysis of cross section and panel data. MIT press. 